\title{
Extension of a Linear Behaviour Model of Shape Memory Alloys for Finite Strain Studies
}

\author{
S. Moyne, M.L. Boubakar and C. Lexcellent \\ Laboratoire de Mécanique Appliquée R. Chaléat, URA 004 du CNRS, 26 chemin de l'Épitaphe, \\ 25030 Besançon cedex, France
}

\begin{abstract}
Within a thermodynamical approach associated to the classical local state postulate, a constitutive macroscopic model, named $R_{L}$ model [1][2], has been built in order to predict the pseudoelastic behaviour of shape memory alloys (SMA). This behaviour occurs with a martensitic transformation. The volume fraction of martensite $z$ is taken as internal variable and the evolution kinetics of $\mathrm{z}$ are derived from metallurgical considerations. In its existing form, the $R_{L}$ model is only valid for small strain calculations. In this paper, an extension is proposed for finite tranformations. Using a formulation in a rotating frame, the obtained model is closed form of the original one. For numerical calculations of NiTi thin films, a stress computation algorithm is also presented. It consists on two steps, the first one is an elastic prediction and the second, a possible pseudoelastic correction. The correction follows an implicit Euler-backward method. To ensure the convergence of the Newton scheme for FE simulation, a consistent tangent operator is used. The results obtained with the proposed approach are in good agreement with the experiments.
\end{abstract}

\section{INTRODUCTION}

The thermomechanical behaviour of shape memory alloys is associated to a recoverable phase transformation between a parent phase: Austenite and a product phase: Martensite. This transformation occurs without any diffusion along an invariant plane $(\mathrm{P})$. If we note ge the macroscopic displacement due to the phase transformation, ge (fig.1) is obtained from an axial deformation with respect to the perpendicular direction $\mathbf{n}$ to $(\mathrm{P})$, and a shear deformation with respect to the parallel direction $\mathbf{d}$. A vector attached to the plane $(P)$ is neither deformed nor rotated. It preserves its initial lenght and its initial orientation.

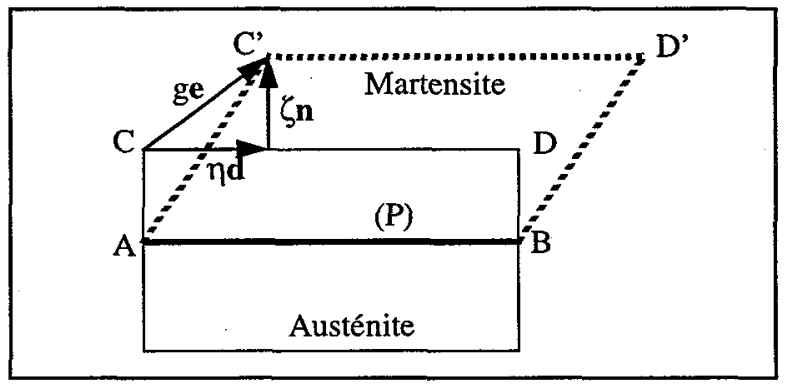

Figure 1: The austenite $A B C D$ is transformed into martensite $A B C$ ' $D$ '

This kind of transformation leads to great recoverable deformations in comparison with those developped by conventional metallic alloys. In general, the monocristallin SMA can support deformations of $10 \%$. In this paper, a kinematics model is proposed to study the SMA in finite deformations. Based on the theory of director frames of Mandel [3], this kinematics leads to the definition of a particular oriented configuration. In this configuration the thermodynamical approach remains consistent and a resulting objective constitutive model is of the form of the one developped in a small strain context [1]. To define an accurate and reliable stress computation algorithm taking into account of the specificity of the martensitic transformation within a finite element simulation, a numerical scheme including an elastic prediction and a pseudoelastic correction can be naturally used. The elastic prediction insures the incremental objectivity 
and a second order accuracy. The correction stage is based on an Euler-backward method and leads to solve a non-linear equation using a local Newton method. Consistency and stability of the proposed scheme are insured. To preserve the convergence of the global Newton method, a consistent tangent operator is used for the calculations. A set of numerical results are presented in cases of the stretching of a bulky structure and NiTi thin films. The accuracy of the solutions and especially the good agreement with experimental results show the efficiency of the proposed approach.

\section{GEOMETRY AND KINEMATICS}

Based on Mandel's approach [3], the following decomposition of the deformation gradient $\mathbf{F}$ is introduced:

$$
\mathbf{F}=\mathbf{V}^{\mathbf{e}} \mathbf{q} \mathbf{F}^{\mathbf{M}}
$$

The tensor $\mathbf{F}^{\mathrm{M}}$ is the gradient of the martensitic transformation from the initial configuration to a particular oriented configuration $\mathrm{C}_{\mathrm{q}}$. In this configuration, a triad of director vectors attached to the material substructure preserves its initial orientation in reference to a fixed cartesian coordinate system. To define the evolution of these vectors during the transformation, an orthogonal tensor $\mathbf{q}$ $(\mathbf{q q}=\mathbf{I} ; \operatorname{det} \mathbf{q}=1)$ is used; a new oriented configuration is then obtained from which a pure elastic deformation $\mathbf{V}^{\mathbf{e}}$ is finally applied. In the case of SMA, a preferred director frame can be defined along the invariant plane $(\mathrm{P})$. During the phase transformation, a vector attached to $(\mathrm{P})$ preserves its initial lenght and its initial orientation.

- Assuming that elastic strains are small $\left(\mathbf{V}^{\mathrm{e}}=\mathbf{I}+\varepsilon^{\mathrm{e}} ;\left\|\varepsilon^{\mathrm{e}}\right\| \ll 1\right)$, the velocity gradient $\mathbf{L}=\dot{\mathbf{F}} \mathbf{F}^{-1}$ leads to the following decompositions of the strain rate tensor $\mathbf{D}$ and of the material spin tensor $\mathbf{W}$ :

$$
\begin{aligned}
& \mathbf{D}=\mathbf{D}^{\mathrm{e}}+\mathbf{D}^{\mathrm{M}}=\mathrm{D}_{\mathbf{q}} \varepsilon^{\mathrm{e}}+\mathbf{q}\left(\dot{\mathbf{F}}^{\mathrm{M}} \mathbf{F}^{\mathrm{M}-1}\right)^{\mathrm{S}} \mathbf{q}^{\mathrm{T}} \\
& \mathbf{W}=\dot{\mathbf{q}} \mathbf{q}^{\mathrm{T}}+\mathbf{W}^{\mathrm{M}}=\dot{\mathbf{q}} \mathbf{q}^{\mathrm{T}}+\mathbf{q}\left(\dot{\mathbf{F}}^{\mathrm{M}} \mathbf{F}^{\mathrm{M}-1}\right)^{\mathrm{A}} \mathbf{q}^{\mathrm{T}}
\end{aligned}
$$

$\mathrm{D}_{\mathrm{q}} \varepsilon^{\mathrm{e}}=\dot{\varepsilon}^{\mathrm{e}}+\varepsilon^{\mathrm{e}}\left(\dot{\mathbf{q}} \mathbf{q}^{\mathrm{T}}\right)-\left(\dot{\mathbf{q}} \mathbf{q}^{\mathrm{T}}\right) \varepsilon^{\mathrm{e}}$ represents the rotational derivative of $\varepsilon^{\mathrm{e}}$ with respect to the rotation $\mathbf{q}$.

Equation (2.a) expresses the additive partition of the strain rates in an elastic part $\mathbf{D}^{\mathbf{e}}$ and in a part $\mathbf{D}^{\mathbf{M}}$ associated to the martensitic transformation. If these quantities are transported in the configuration $\mathrm{C}_{\mathrm{q}}$, we obtain:

$$
\dot{\varepsilon}_{\mathrm{q}}^{\mathrm{e}}=\dot{\mathbf{d}}_{\mathrm{q}}-\dot{\mathbf{d}}_{\mathrm{q}}^{\mathrm{M}}
$$

with $\dot{\varepsilon}_{q}^{\mathrm{e}}=\frac{\mathrm{d}}{\mathrm{dt}}\left(\mathbf{q}^{\mathrm{T}} \varepsilon^{\mathrm{e}} \mathbf{q}\right), \dot{\mathbf{d}}_{\mathrm{q}}=\mathbf{q}^{\mathrm{T}} \mathbf{D q}=\frac{\mathrm{d}}{\mathrm{dt}}\left(\mathbf{q}^{\mathrm{T}} \mathbf{d q}\right)$ and $\dot{\mathbf{d}}_{\mathrm{q}}^{\mathrm{M}}=\mathbf{q}^{\mathrm{T}} \mathbf{D}^{\mathrm{M}} \mathbf{q}=\frac{\mathrm{d}}{\mathrm{dt}}\left(\mathbf{q}^{\mathrm{T}} \mathbf{d}^{\mathrm{M}} \mathbf{q}\right)$. d and $\mathbf{d}^{\mathrm{M}}$ can be interpreted as strain tensors associated with the strain rates $\mathbf{D}$ and $\mathbf{D}^{\mathbf{M}}$ with respect to the ration $q$. The equation (2.b) gives a relationship between the material spin $W$, the $\operatorname{spin} \mathbf{W}^{M}$ of the medium with respect to the director vectors and their spin $\dot{\mathbf{q}} \mathbf{q}^{\mathrm{T}}$. That permits to take into account an oriented substructure. In the case of isotropic alloys, $\mathbf{W}^{\mathbf{M}}=0$ and $\dot{\mathbf{q}} \mathbf{q}^{\mathbf{T}}=\mathbf{W}$. We restrict our study to this case.

\section{CONSTITUTIVE MODEL}

We assume that the state of the material is completely definied in the configuration $\mathrm{C}_{\mathrm{q}}$ by the temperature $T$, the volume fraction of martensite $z$ and the elastic deformation tensor $\varepsilon_{q}^{e}=q^{T} \varepsilon^{e} \mathbf{q}$. The elastic deformations are assumed to be the same for each phase. The specific free energy of the considered twophases system is chosen in the following form: 


$$
\phi\left(\varepsilon_{\mathrm{q}}^{\mathrm{e}}, \mathrm{T}, \mathrm{z}\right)=(1-\mathrm{z}) \phi_{1}\left(\varepsilon_{\mathrm{q}}^{\mathrm{e}}, \mathrm{T}\right)+\mathrm{z} \phi_{2}\left(\varepsilon_{\mathrm{q}}^{\mathrm{e}}, \mathrm{T}\right)+\mathrm{z}(1-\mathrm{z}) \phi_{\mathrm{jt}}(\mathrm{T})
$$

where $\mathrm{z}$ is the volume of the product phase (martensite) and (1-z) the volume of the parent phase (austenite). The quantity $\mathrm{z}(1-\mathrm{z}) \phi_{\mathrm{it}}(\mathrm{T})\left(\phi_{\mathrm{it}}(\mathrm{T})=\overline{\mathrm{u}}_{0}-\mathrm{T} \overline{\mathrm{s}}_{0} ; \overline{\mathrm{u}}_{0}\right.$ and $\overline{\mathrm{s}}_{0}$ are constants) represents the interaction effects between the two phases, typically the incompatibilities between deformations. This quantity is equal to zero when we have one phase. The specific energy $\phi_{\alpha}(\alpha=1,2)$ of each phase is given by:

$$
\phi_{\alpha}\left(\varepsilon_{\mathrm{q}}^{\mathrm{e}}, \mathrm{T}\right)=\mathrm{u}_{0 \alpha}-\mathrm{Ts}_{0 \alpha}+\frac{1}{2 \rho_{0}} \varepsilon_{\mathrm{q}}^{\mathrm{e}}: \mathbf{C}^{\mathrm{e}}: \varepsilon_{\mathrm{q}}^{\mathrm{e}}+\mathrm{c}_{\mathrm{v}}\left(\mathrm{T}-\mathrm{T}_{0}-\mathrm{TLn} \frac{\mathrm{T}}{\mathrm{T}_{0}}\right)
$$

$u_{0 \alpha}$ is the internal energy and $s_{0 \alpha}$ the entropy of the phase $\alpha$ at the reference temperature $T_{0}$. We assume that each phase has the same elastic constants represented by the fourth order tensor $\mathbf{C}^{\mathfrak{e}}$, the same specific heat $c_{v}$ and the same mass density $\rho_{0}$. Using (5) in (4), we finally obtain:

$$
\phi\left(\varepsilon_{\mathrm{q}}^{\mathrm{e}}, \mathrm{T}, \mathrm{z}\right)=\left(\mathrm{u}_{01}-\mathrm{Ts} s_{01}\right)-\mathrm{z} \Pi_{0}^{\mathrm{f}}+\frac{1}{2 \rho_{0}} \varepsilon_{\mathrm{q}}^{\mathrm{e}}: \mathrm{C}^{\mathrm{e}}: \varepsilon_{\mathrm{q}}^{\mathrm{e}}+\mathrm{c}_{\mathrm{v}}\left(\mathrm{T}-\mathrm{T}_{0}-\mathrm{TLn} \frac{\mathrm{T}}{\mathrm{T}_{0}}\right)+\mathrm{z}(1-\mathrm{z}) \phi_{\mathrm{it}}(\mathrm{T})
$$

$\Pi_{0}^{\mathrm{f}}(\mathrm{T})=\left(\mathrm{u}_{01}-\mathrm{u}_{02}\right)-\mathrm{T}\left(\mathrm{s}_{01}-\mathrm{s}_{02}\right)=\Delta \mathrm{u}_{0}-\mathrm{T} \Delta \mathrm{s}_{0}$ constitutes the driving force of the martensitic transformation for a thermal action.

To establish the state laws and to define the transition phase criterion, we work within the thermodynamic approach of the irreversible processes. Taking account of (2.a), (3) and (6), the ClausiusDuhem inequality takes the following form:

$$
\left(\sigma_{\mathrm{q}}-\rho_{0} \frac{\partial \phi}{\partial \varepsilon_{\mathrm{q}}^{\mathrm{e}}}\right): \dot{\varepsilon}_{\mathrm{q}}^{\mathrm{e}}-\rho_{0}\left(\mathrm{~s}+\frac{\partial \phi}{\partial \mathrm{T}}\right) \dot{\mathrm{T}}+\sigma_{\mathrm{q}}: \dot{d}_{\mathrm{q}}^{\mathrm{M}}-\rho_{0} \frac{\partial \phi}{\partial \mathrm{z}} \dot{z} \geq 0
$$

$\mathrm{s}$ is the specific entropy of the system. We deduce:

$$
\sigma_{\mathrm{q}}=\rho_{0} \frac{\partial \phi}{\partial \varepsilon_{\mathrm{q}}^{\mathrm{e}}} \quad \mathrm{s}=-\frac{\partial \phi}{\partial \mathrm{T}} \quad \sigma_{\mathrm{q}}: \dot{\mathbf{d}}_{\mathrm{q}}^{\mathrm{M}}-\rho_{0} \frac{\partial \phi}{\partial \mathrm{z}} \dot{\mathrm{z}} \geq 0
$$

with:

$$
\begin{gathered}
\frac{\partial \phi}{\partial \mathrm{z}}=-\Pi_{0}^{\mathrm{f}}(\mathrm{T})+(1-2 \mathrm{z}) \phi_{\mathrm{it}}, \quad \frac{\partial \phi}{\partial \varepsilon_{\mathrm{q}}^{\mathrm{e}}}=\frac{1}{\rho_{0}} \mathbf{C}^{\mathrm{e}}: \varepsilon_{\mathrm{q}}^{\mathrm{e}} \\
\frac{\partial \phi}{\partial \mathrm{T}}=-\mathrm{s}_{01}+\mathrm{z} \Delta \mathrm{s}_{0}-\mathrm{c}_{\mathrm{v}} \operatorname{Ln} \frac{\mathrm{T}}{\mathrm{T}_{0}}+\mathrm{z}(1-\mathrm{z}) \frac{\partial \phi_{\mathrm{it}}}{\partial \mathrm{T}}, \quad \sigma_{\mathrm{q}}=\mathbf{q}^{\mathrm{T}} \sigma \mathbf{q}
\end{gathered}
$$

With electrical measurments "in situ" (giving the martensite fraction evolution) during pseudoelastic tensile tests, it has been established [4] the proportionality between the deformation $\mathbf{d}_{q}{ }^{M}$ associated with the martensitic transformation and the volume fraction of martensite $\mathrm{z}$. We use here this propriety between the rates:

$$
\dot{\mathbf{d}}_{\mathrm{q}}^{\mathrm{M}}=\sqrt{\frac{3}{2}} \gamma \frac{\mathbf{P}: \sigma_{\mathrm{q}}}{\sqrt{\sigma_{\mathrm{q}}: \mathbf{P}: \sigma_{\mathrm{q}}}} \dot{\mathbf{z}}=\frac{3}{2} \gamma \frac{\mathbf{P}: \sigma_{\mathrm{q}}}{\bar{\sigma}_{\mathrm{q}}} \dot{\mathbf{z}}
$$

The fourth order tensor $\mathbf{P}$ is the deviatoric projection operator and $\gamma$ is the total pseudoelastic uniaxial strain obtained with tensile test. Using this relation in the dissipation (8.c), it comes:

$$
\Pi^{\mathrm{f}}\left(\sigma_{\mathrm{q}}, \mathrm{T}, \mathrm{z}\right) \dot{\mathrm{z}} \geq 0
$$


$\Pi^{f}\left(\sigma_{q}, T, z\right)=\gamma \bar{\sigma}_{q}+\rho_{0} \Pi_{0}^{f}(T)-\rho_{0}(1-2 z) \phi_{i t}$ is then the thermodynamical force of the phase transformation. This inequality leads to the following transformation conditions:

$$
(A \rightarrow M): \quad \Pi^{f}\left(\sigma_{q}, T, z\right) \geq 0, \dot{z} \geq 0 \quad(M \rightarrow A): \Pi^{f}\left(\sigma_{q}, T, z\right) \leq 0, \quad \dot{z} \leq 0
$$

To specify the kinetic equations of phase transformations we presume that there exists two functions $\psi_{\alpha}$ $(\alpha=1,2)$ such that an active process of parent phase decomposition $A \rightarrow M$ can proceed only when $\psi_{1}=$ cte $\left(\mathrm{d} \psi_{1}=0\right)$ and an active process of martensite decomposition $M \rightarrow A$ can proceed only if $\psi_{2}=\operatorname{cte}\left(d \psi_{2}=0\right)$ :

$$
\psi_{1}=\Pi^{\mathrm{f}}\left(\sigma_{\mathrm{q}}, \mathrm{T}, \mathrm{z}\right)-\mathrm{k}_{1}(\mathrm{z}) \quad \psi_{2}=-\Pi^{\mathrm{f}}\left(\sigma_{\mathrm{q}}, \mathrm{T}, \mathrm{z}\right)+\mathrm{k}_{2}(\mathrm{z})
$$

For special choice of the functions $\mathrm{k}_{\alpha}(\mathrm{z})$, the classical relationships for transformation kinetics of metallurgists as in [5] are recovered:

$$
\begin{array}{ll}
\mathrm{k}_{1}(\mathrm{z})=-\frac{1}{\mathrm{a}_{1}}\left(\Delta \mathrm{s}_{0}-(1-2 \mathrm{z}) \overline{\mathrm{s}}_{0}\right) \operatorname{Ln}(1-\mathrm{z})+2 \phi_{\mathrm{it}}\left(\mathrm{M}_{\mathrm{s}}^{0}\right) \mathrm{z} & \mathrm{a}_{1}=\frac{-\operatorname{Ln}(0.01)}{\mathrm{M}_{\mathrm{s}}^{0}-\mathrm{M}_{\mathrm{f}}^{0}} \\
\mathrm{k}_{2}(\mathrm{z})=\frac{1}{\mathrm{a}_{2}}\left(\Delta \mathrm{s}_{0}-(1-2 \mathrm{z}) \overline{\mathrm{s}}_{0}\right) \operatorname{Lnz}-2 \phi_{\mathrm{it}}\left(\mathrm{A}_{\mathrm{s}}^{0}\right)(1-\mathrm{z}) & \mathrm{a}_{2}=\frac{-\operatorname{Ln}(0.01)}{\mathrm{A}_{\mathrm{s}}^{0}-\mathrm{A}_{\mathrm{f}}^{0}}
\end{array}
$$

$\mathrm{M}_{\mathrm{s}}^{0}$ and $\mathrm{A}_{\mathrm{s}}^{0}$ are, respectively, the temperatures of the martensitic and austenitic transformation start for $\sigma_{\mathrm{q}}=0 . \mathrm{M}_{\mathrm{f}}^{0}$ and $\mathrm{A}_{\mathrm{f}}^{0}$ are the same temperatures at the end of the transformations.

The consistency equations $d \psi_{\alpha}=0$ permit to obtain the kinetic equations:

$$
\mathrm{d} \psi_{1}=\frac{\partial \Pi^{\mathrm{f}}}{\partial \bar{\sigma}_{\mathrm{q}}} \mathrm{d} \bar{\sigma}_{\mathrm{q}}+\frac{\partial \Pi^{\mathrm{f}}}{\partial \mathrm{T}} \mathrm{dT}+\left(\frac{\partial \Pi^{\mathrm{f}}}{\partial \mathrm{z}}-\frac{\partial \mathrm{k}_{1}}{\partial \mathrm{z}}\right) \mathrm{dz}=0
$$

For isothermal pseudoelastic test $(\mathrm{dT}=0)$, the expression of $\Pi^{\mathrm{f}}$ gives:

$$
\frac{\partial \Pi^{\mathrm{f}}}{\partial \bar{\sigma}_{\mathrm{q}}}=\frac{\gamma}{\rho_{0}} \quad \frac{\partial \Pi^{\mathrm{f}}}{\partial \mathrm{z}}-\frac{\partial \mathrm{k}_{1}}{\partial \mathrm{z}}=\mathrm{H}_{\sigma}^{1}
$$

with:

$$
H_{\sigma}^{1}=2 \bar{s}_{0}\left(T-M_{s}^{0}\right)+\frac{\Delta s_{0}}{a_{1}(1-z)}+\frac{2 \bar{s}_{0}}{a_{1}}\left(\frac{2 z-1}{2(1-z)}-\operatorname{Ln}(1-z)\right)
$$

at least:

$$
\mathrm{dz}^{\mathrm{A} \rightarrow \mathrm{M}}=\frac{\gamma}{\rho_{0} \mathrm{H}_{\sigma}^{1}} \mathrm{~d} \bar{\sigma}_{\mathrm{q}}
$$

In the same way, we obtain

$$
\mathrm{dz} \mathrm{z}^{\mathrm{M} \rightarrow \mathrm{A}}=\frac{\gamma}{\rho_{0} \mathrm{H}_{\sigma}^{2}} \mathrm{~d} \bar{\sigma}_{\mathrm{q}}
$$

with:

$$
H_{\sigma}^{2}=2 \bar{s}_{0}\left(T-A_{s}^{0}\right)+\frac{\Delta s_{0}}{a_{2} z}+\frac{2 \bar{s}_{0}}{a_{2}}\left(\frac{2 z-1}{2 z}-\operatorname{Ln}(z)\right)
$$




\section{STRESS CALCULATION ALGORITHM}

The simple form of constitutive equations written in the configuration $\mathrm{C}_{\mathrm{q}}$ allows, within the scope of the incremental method, to calculate for each displacement increment $\Delta \mathbf{u}$ the stress state in both steps. Firstly an elastic prediction step then a possible pseudoelastic correction one.

\subsection{Elastic prediction step}

In this first step, the behaviour is supposed to be elastic. The equation (8.a) is integrated over the time interval $\left[t_{n}, t_{n+1}\right]$ (subscripts $n$ and $n+1$ correspond to values at the beginning and at the end of the loading step under consideration) to give the trial stress state. Using the generalized mid-point rule, we obtain:

$$
\left(\sigma_{\mathrm{q}}\right)_{\mathrm{n}+1}^{\text {trial }}=\left(\sigma_{\mathrm{q}}\right)_{\mathrm{n}}+\mathbf{C}^{\mathrm{e}} \Delta \mathrm{t}\left(\mathbf{d}_{\mathrm{q}}\right)_{\mathrm{n}+\alpha} \quad \Delta \mathrm{t}=\mathrm{t}_{\mathrm{n}+1}-\mathrm{t}_{\mathrm{n}}
$$

The components of $\Delta t\left(d_{q}\right)_{n+\alpha}$ in the configuration $C_{q}$ with reference to a rotating frame are those of $\Delta t D_{n+\alpha}$ (D is the strain rate tensor) with reference to the same frame in the intermediate configuration $\mathrm{C}_{\mathrm{n}+\alpha}$. If we use a linear interpolation for the geometry, $\Delta \mathrm{tD} \mathrm{D}_{\mathrm{n}+\alpha}$ can be approximated by [6]:

$$
\Delta t D_{n+\alpha}=\left[\frac{\partial \Delta \mathbf{u}}{\partial x_{n+\alpha}}\right]^{s}
$$

with:

$$
\mathbf{x}_{\mathrm{n}+\alpha}=(1-\alpha) \mathbf{x}_{\mathrm{n}}+\alpha \mathbf{x}_{\mathrm{n}+1} \quad \alpha \in[0,1]
$$

In particular, the value $\alpha=1 / 2$ insure the incremental objectivity and a second order accuracy.

\subsection{Pseudoelastic correction step}

If the yield function (13.a) becomes positive or (13.b) negative after the prediction step, a correction is made from (2), (8.a) and (10) using an implicit Euler-backward method:

$$
\left(\sigma_{\mathrm{q}}\right)_{\mathrm{n}+1}=\mathbf{G}^{-1}(\Delta \mathrm{z})\left(\sigma_{\mathrm{q}}\right)_{\mathrm{n}+1}^{\text {trial }} \quad \mathbf{G}^{-1}(\Delta \mathrm{z})=\mathbf{I}-3 \mathrm{G} \gamma \frac{\Delta \mathrm{z}}{\left(\bar{\sigma}_{\mathrm{q}}\right)_{\mathrm{n}+1}^{\text {trial }}} \mathbf{P}
$$

$\Delta \mathrm{z}$ is obtained from the non-linear equation (18) when $\mathrm{A} \rightarrow \mathrm{M}$ or from (19) when $\mathrm{M} \rightarrow \mathrm{A}$.

\section{NUMERICAL RESULTS}

The proposed approach is used here to simulate the stretching of a NiTi alloy in the case of bulky structure and thin film [7]. The thermodynamical constants are given in table 1. The transformation temperatures of the considered alloy are $A_{s}=262^{\circ} \mathrm{K}$ and $M_{s}=297^{\circ} \mathrm{K}$. The results obtained for a bulky structure are shown figure 2. It appears that the behaviour is well predicted in comparison with the experimental results. Meanwhile, the value of $A_{s}$ is not in agreement with the experiment. For thin films (thickness $=7.1 \mu \mathrm{m}$ ), the results of the simulation are shown figure 3 .

\begin{tabular}{|c|c|c|c|c|c|c|c|}
\hline $\begin{array}{c}\mathrm{a}_{1} \\
\left({ }^{\circ} \mathrm{K}^{-1}\right)\end{array}$ & $\begin{array}{c}\mathrm{a}_{2} \\
\left({ }^{\circ} \mathrm{K}^{-1}\right)\end{array}$ & $\gamma$ & $\begin{array}{c}\rho_{0} \\
\left(\mathrm{Kg}^{3}\right)\end{array}$ & $\begin{array}{c}\Delta \mathrm{u}_{0} \\
\left(\mathrm{~J} . \mathrm{Kg}^{-1}\right)\end{array}$ & $\begin{array}{c}\Delta \mathrm{s}_{0} \\
\left(\mathrm{~J} . \mathrm{Kg}^{-1}{ }^{\circ} \mathrm{K}^{-1}\right)\end{array}$ & $\begin{array}{c}\overline{\mathrm{u}}_{0} \\
\left(\mathrm{~J} . \mathrm{Kg}^{-1}\right)\end{array}$ & $\begin{array}{c}\overline{\mathrm{s}}_{\mathrm{P}} \\
\left(\mathrm{J} . \mathrm{Kg}^{-1} \mathrm{~K}^{-1}\right)\end{array}$ \\
\hline \hline 0.7 & 0.07 & 0.035 & 6500 & 10956 & 37.2 & 2848 & 6.35 \\
\hline
\end{tabular}




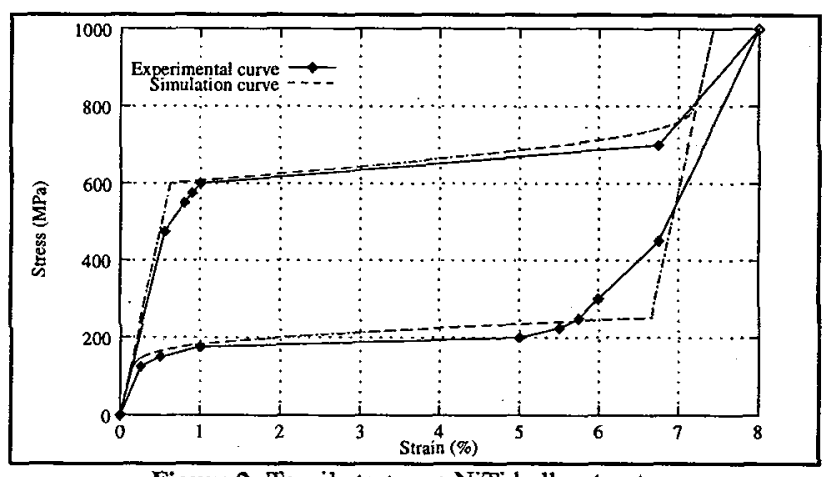

Figure 2: Tensile test on a NiTi bulky structure

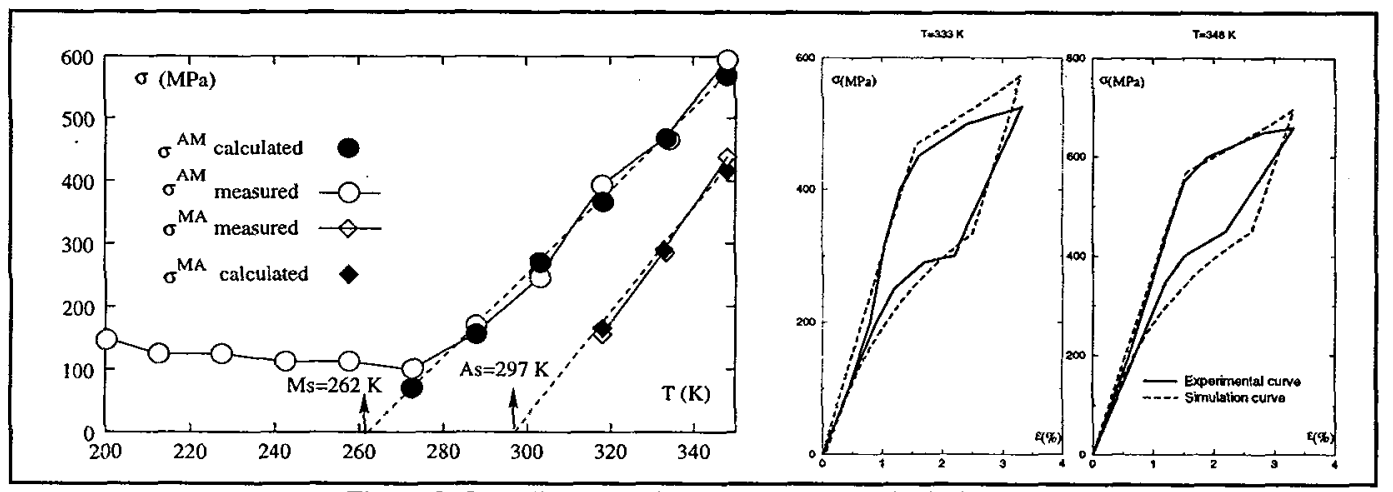

Figure 3: State diagram and tensile test on a NiTi thin film [7]

\section{CONCLUSION}

Within the developpement of a finite element code for the behaviour simulation of adaptive structures, an extension of $R_{L}$ model to the case of finite strains is proposed. The constitutive equations have the same form of those developped for small strain calculations. That leads to built a stress computation algorithm based on the predictor-corrector scheme combined with a local Newton method. The accuracy of the solutions and especially the good agreement with experimental results show the efficiency of the proposed approach. This approach is now used with a new class of shell elements [8] to simulate bending and bulging tests of NiTi thin films. The proposed stress calculation algorithm takes into account of the formulation of the shell elements and especially of the mixed interpolation used in order to avoid transverse shear locking.

\section{References}

[1] Raniecki B. et Lexcellent C., Eur. J. of Mech. A/Solids, 13(1) (1994), 21-50.

[2] Raniecki B., Lexcellent C. et Tanaka K., Arch. Mech., 44 (1992), 262-288.

[3] Mandel J., Int. J. Solids Structures, Vol. 9 (1973), 725-740.

[4] Vacher P. et Lexcellent C., Proceedings of ICM VI, Vol. 6 (1991), 231-236.

[5] Koistinen D. P. and Marburger R. E., Acta. Meta., 7 (1959), 59-69.

[6] Hughes T. J. R. and Winget J., Int. J. for Num. Meth. in Eng., Vol. 15 (1980), 1862-1867.

[7] Li Hou and Grummon D. S., Scripta Matallurgica et Materialia, 33(6) (1995), 989-995.

[8] Boubakar M. L., Boulmane L. and Gelin J. C., Engineering Computations, Vol, 13 (1996), 143-171. 